\title{
Dissipative energy as an indicator of material microstructural evolution
}

\author{
N. Connesson ${ }^{1}$, F. Maquin ${ }^{1}$, and F. Pierron ${ }^{1}$ \\ Laboratoire de mécanique et procédés de fabrication (LMPF), Arts et Metiers ParisTech, rue St Do- \\ minique, BP 508, 51006 Châlons-en-Champagne cedex, France
}

\begin{abstract}
In this study, the material microstructure evolution has been studied thanks to two indicators: the cumulated plastic strain and the energy dissipation due to internal friction under cyclic loading. An experimental procedure has been designed to underline the variations of the dissipative energy due to cold work on a DP600 specimen. The results showed that the dissipative energy increases with the plastic strain and can be used as an indicator of material microstructural evolution.
\end{abstract}

\section{Introduction}

Fatigue characterization is a time consuming and expensive operation commonly undertaken in industria. The development of many theories and experimental measurement technics is thus of first interest to provide faster fatigue limit estimation methods. Thus, with the development of infrared cameras, some authors proposed experimental methods to estimate the fatigue limits based on the material temperature increase under cyclic loading $[1,2]$. Yet, the physical explanation of such methods is not fully understood, and heat dissipation phenomena needs to be more thoroughly studied.

The thermomechanical sources of materials can be dissociated into two main phenomena, such as the thermomechanical coupling and the dissipative energy. The dissipative energy, responsible for the mean temperature increase of the specimen under harmonic loading, can be experimentally measured by solving a heat balance equation [3]. An experimental method to finely measure the dissipative energy is proposed in [4] and is used in this study.

The dissipative energy phenomena is related to internal friction mechanisms [5]: under loading, the material defects (inclusion, dislocations, etc) are moving in the material lattice and dissipate energy, the main part of dissipated energy being attributed to dislocation movements. It seems thus that the dissipative energy should depend on the material microstructure such as the mobile dislocation density, their spatial repartition and interactions, the lattice friction coefficient etc. In fewer words, the dissipative energy should be a good experimental criteria to monitor the material microstructural evolution.

Moreover, it is well known that loading history has a direct impact on the material microstructure. Depending on the applied loading, the dislocations move, extend, multiply [6,7], or create specific spatial structures [8-11] such as cells, persistent slip bands etc. The dislocation density usually increase with the plastic strain.

In this study, it is thus chosen to observe the correlation between the dissipative energy and the plastic strain, both phenomena related to the microstructural dislocation structure.

Few works have been made to analyse the dissipative energy evolution with the cold work. The temperature versus alternate stress curve has been studied in $[12,13]$ on different cold worked specimens. Yet, as the materials were loaded with $R_{\sigma}=\frac{\sigma_{\min }}{\sigma_{\max }}=-1$ (where $\sigma_{\min }$ and $\sigma_{\max }$ are respectively the minimum and maximal stress), heat dissipation was due both to internal friction and macroscopic plasticity.

This is an Open Access article distributed under the terms of the Creative Commons Attribution-Noncommercial License 3.0, which permits unrestricted use, distribution, and reproduction in any noncommercial medium, provided the original work is properly cited. 
In this work, these two dissipative energies have been dissociated and attention has been focussed only on the internal friction heat dissipation. Thus, in the rest of this study, internal friction heat dissipation will be simply referred as "heat dissipation" or "dissipative energy".

This paper present thus an experimental measurement of the dissipative energy variation along with the uniaxial plastic strain of a dual phase steel (DP600). The idea is that such experimental data may help to better understand heat dissipation phenomena and provide some data to temperature increase predictive models.

\section{Experimental procedure and results}

This study has been performed on a specimen machined in a $2 \mathrm{~mm}$ thick steel plate of DP600 steel which geometry is presented in Figure 1. The dissipative energy and plastic strain in the observed area $\Omega_{s p}$ have been monitored with the experimental setup presented hereafter.

Plastic strain measurement. The strain has been measured with a $350 \Omega$ strain gauge (Vishay, CEA06-250UW-350) connected to a quarter Wheatstone Bridge. This strain gage was bonded on one side of the specimen in the loading direction. In all this study, plastic strain has been measured while no loading was applied to the specimen.

Dissipative energy measurement. The dissipative energy is measured using an infrared CEDIP Jade camera by applying the experimental procedure proposed in [4]: during a dissipative energy measurement, the specimen is cyclically loaded and the temperature field of the observed area $\Omega_{s p}$ is acquired. The dissipatives sources are estimated using a local energy balance which general shape is:

$$
\rho C\left(\frac{\partial \theta}{\partial t}-\left[\frac{\partial \theta}{\partial t}\right]_{\left(t=0^{-}\right)}\right)-k \Delta_{2} \theta+\rho C \frac{\theta}{\tau_{t h}^{2 D}}=d_{1}+S_{t h}
$$

where $\rho$ is the material density, $C$ the calorific capacity, $k$ the thermal conductivity and $\Delta_{2}$ the laplacien operator. $\theta$ is here the local temperature variation measured with the infrared camera. This equation gives thus the local dissipative sources $d_{1}$ and the sources due to the thermoelastic coupling $S_{t h}$ by estimating the energy storage by temperature increase $\left(\rho C\left(\frac{\partial \theta}{\partial t}-\left[\frac{\partial \theta}{\partial t}\right]_{\left(t=0^{-}\right.}\right)\right)$, energy losses by conduction $\left(k \Delta_{2} \theta\right)$ and energy losses by convection and radiation $\left(\rho C \frac{\theta}{\tau_{t h}^{2 D}}\right) \cdot \tau_{t h}^{2 D}$ is thus a time constant characterizing the convection and radiation losses. This equation is then integrated over the observed area $\Omega_{s p}$ and over each cycle which provides the dissipative energy per cycle $E_{d_{1}}^{i}=\int_{t_{i}}^{t_{i}+1 / f_{L}} \frac{1}{\Omega_{s p}} \int_{\Omega_{s p}} d_{1} d x d y d t$. Here, $f_{L}$ is the loading frequency and $t_{i}$ the beginning of the cycle $i$. In this study, the specimen have been harmonically loaded with $R_{\sigma}=\frac{\sigma_{\min }}{\sigma_{\max }}=0.1$. If the

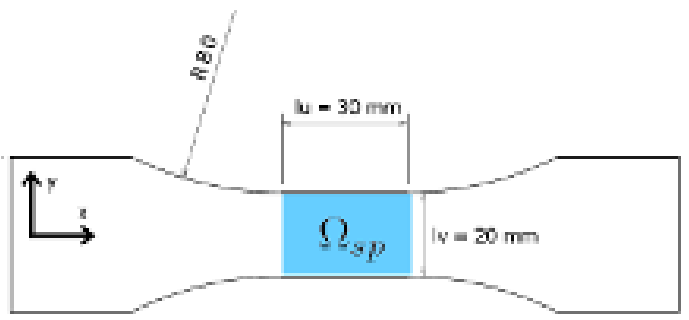


Moreover, as the dissipative energy is constant during these measurement, only the mean of the dissipative energy has been used in this paper. This mean will be noted $E_{d_{1}}^{m}$ in the reste of this paper. A noise level analysis showed that the measurement incertitude of a mean dissipative energy $E_{d_{1}}^{m}$

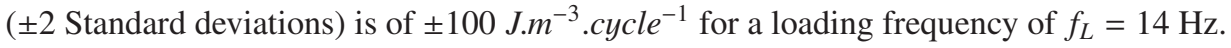

This experimental setup provides thus the plastic strain $\epsilon^{p}$ while the specimen is not loaded and the dissipative energy $E_{d_{1}}^{m}$ under cyclic loading in steady state conditions. Measurements sequences have been organized in this study to monitor the correlation between dissipative energy and plastic strain.

Four measurement sequences at different loading levels (Phase I to IV) have successively been applied to the specimen, each sequence being designed to reach a different goal: the material initial state behaviour is analyzed during Phase I. The material is then plastically strained and its evolution is analyzed during Phase II. Phase III and IV are then performed to monitor the changes in the material dissipative energy due to the previous two phases.

The tests sequences are represented graphically in the upper graph of Figure 2. In this representation, each point represents three successive tests:

- a cyclic loading at the maximum stress $\sigma_{\max }$ (represented by the point ordinate) applied until a steady state is reached,

- then a dissipative energy measurement in the same loading conditions,

- and eventually a plastic strain measurement $\epsilon^{p}$ while the loading is zero.

The cumulated plastic strain $\epsilon^{p}$ at the end of each test is presented in the bottom graph of Figure 2.

Phase I: the maximal stress $\sigma_{\max }$ was increased form 100 to $240 \mathrm{MPa}$ by step of $20 \mathrm{MPa}$. The yield stress at $0.02 \%$ being of $295 \mathrm{MPa}$, the cumulated plastic strain remained small.

Phase II: as in phase I, the maximal stress $\sigma_{\max }$ was increased from 100 to $240 \mathrm{MPa}$ by step of $20 \mathrm{MPa}$. The plastic strain did not change during these first steps since the material accommodated to these stresses during Phase I. Then, the maximal stress $\sigma_{\max }$ was increased from 260 to $440 \mathrm{MPa}$ by step of $20 \mathrm{MPa}$ while some tests were performed at $\sigma_{\max }=240 \mathrm{MPa}$ in-between $\left(\operatorname{dots} D_{2} . . D_{10}\right)$. Plastic strain occurred during this phase as the material adapted itself to the loading. No additional strain has been observed during the in-between tests $D_{i}$. The strain gage bond failed during the test at $\sigma_{\max }=440 \mathrm{MPa}$. No plastic strain measurements were thus available after $D_{8}$.

Phase III and IV: as in Phase II, the maximal stress $\sigma_{\max }$ was increased from 100 to $440 \mathrm{MPa}$ by step of $20 \mathrm{MPa}$.

The dissipative energy $E_{d_{1}}^{m}$ measured during each test is presented versus alternate stress $\sigma_{a}$ and maximal stress $\sigma_{\max }$ in Figure 3. As the measurement incertitude, which is of $\pm 100 \mathrm{~J}_{\mathrm{m}} \mathrm{m}^{-3}$.cycle $\mathrm{e}^{-1}$, is small compared to the scales, no error-bars have been printed in this graph.

The general behavior is that the dissipative energy per cycle $E_{d_{1}}^{m}$ increases with the alternate stress. The dissipative energies during Phase I (Tests up to $A$ ) and the first tests of Phase II (Tests up to $D_{0}$ ) were identical: the plastic strain occurring during Phase I (Figure 2) did not change the dissipative energy behaviour. This result shows thus that the dissipative energy measurement is reproducible on the same specimen if the material is not plastically strained.

The dissipative energy of the tests $A, D_{0} . . D_{10}, C, E$, which were all performed at the same maximal stress $\sigma_{\max }=240 \mathrm{MPa}$ and in steady state behaviour, increase gradually along with the material cumulated plastic strain. Moreover, the dissipative energy during Phase III is always greater than the dissipative energy during Phase II. The material microstructural changes, occurring during Phase II, increase thus progressively the dissipated energy. These results confirms that the dissipated energy is an indicator of the material microstructural state.

Moreover, the dissipative energies during the last test of Phase II and Phase III (Tests $B$ and $D$ ) are identical. The material modifications due to the cumulated plastic strain occurring between the last test of Phase II and Phase III have thus no visible influence on the material dissipative energy behaviour at $\sigma_{\max }=440 \mathrm{MPa}$. Eventually, the measurements achieved during Phase IV point out once again the good reproducibility of this measurement on the same specimen if no plastic strain occurs. Phase III and IV are thus the characterization of the dissipative energy stabilized behaviour: this curve could be the material microstructure "signature". 


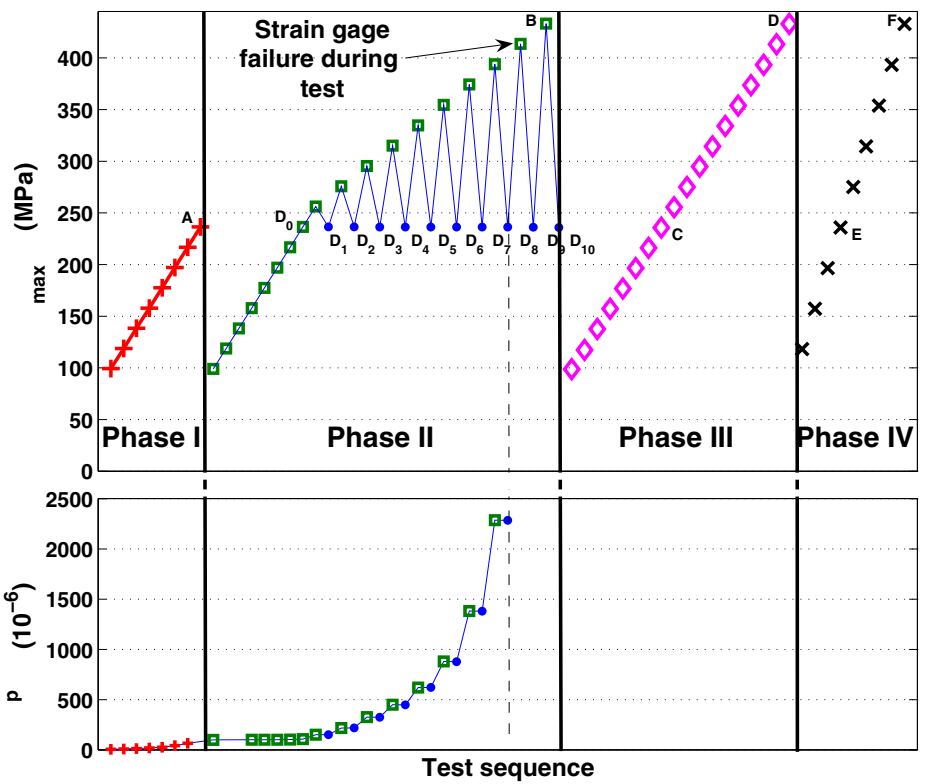

Fig. 2. Maximal stress $\sigma_{\max }$ applied for each test and cumulated plastic strain $\epsilon^{p}$ at the end of each test.

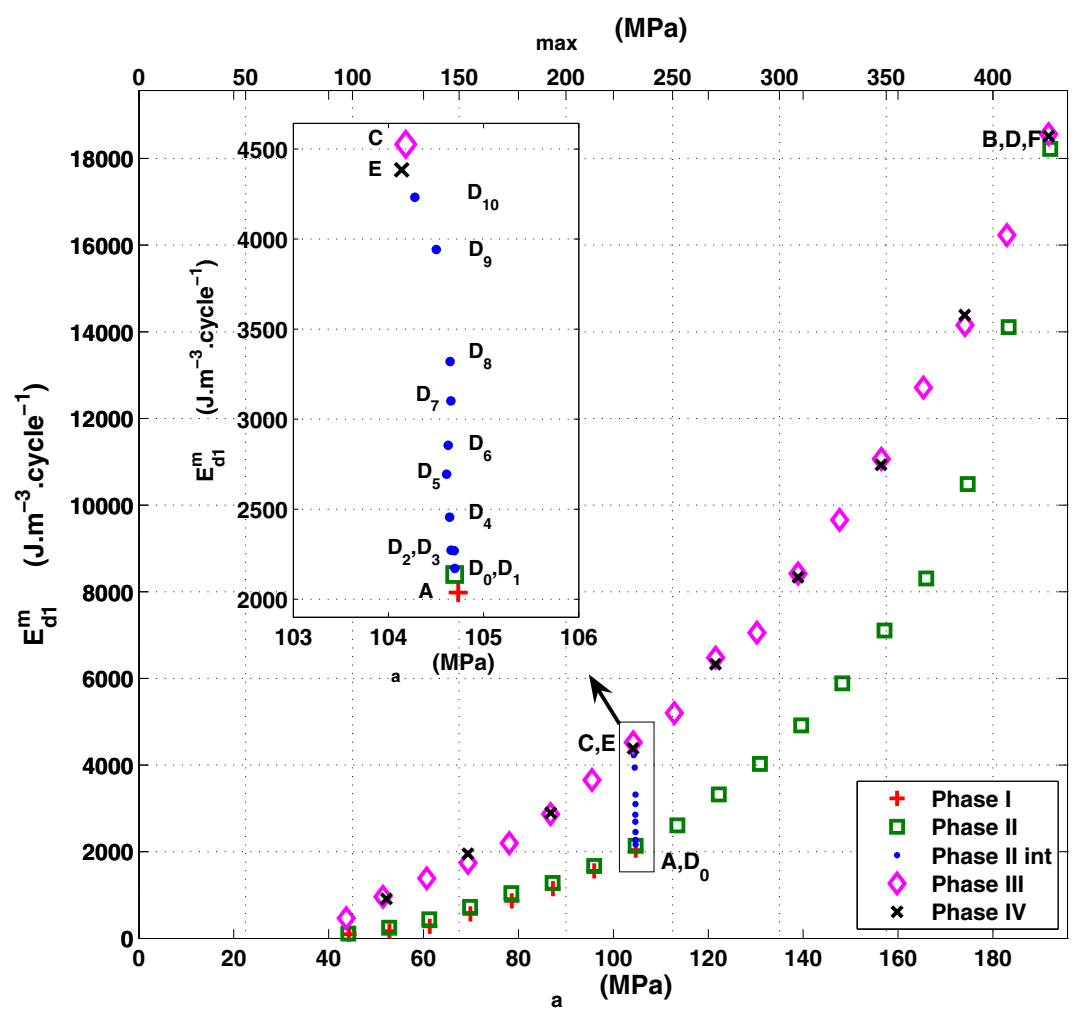

Fig. 3. Dissipative energy measurement $E_{d_{1}}^{m}$ versus alternate stress $\sigma_{a}$ (bottom scale) and maximal stress $\sigma_{\max }$ (top scale). 
It is also possible to present the dissipative energy $E_{d_{1}}^{m}$ measured during the tests $A, D_{0} . . D_{8}$ versus the cumulated plastic strain $\epsilon^{p}$ (Figure 4). The dissipatives energies $E_{d_{1}}^{m}$ of this graph have all been measured with the same experimental settings $\left(\sigma_{a}=104,6 \pm 0.25^{1} \mathrm{MPa}, R_{\sigma}=0,1\right.$, etc.). The only difference from one test to another is thus the material microstructure modification due to cold work. Figure 4 presents thus in a different way that the dissipative energy $E_{d_{1}}^{m}$ increases with the plastic strain. It is yet worth noting that the increase rate of the dissipative energy is greater for small plastic cumulated strain.

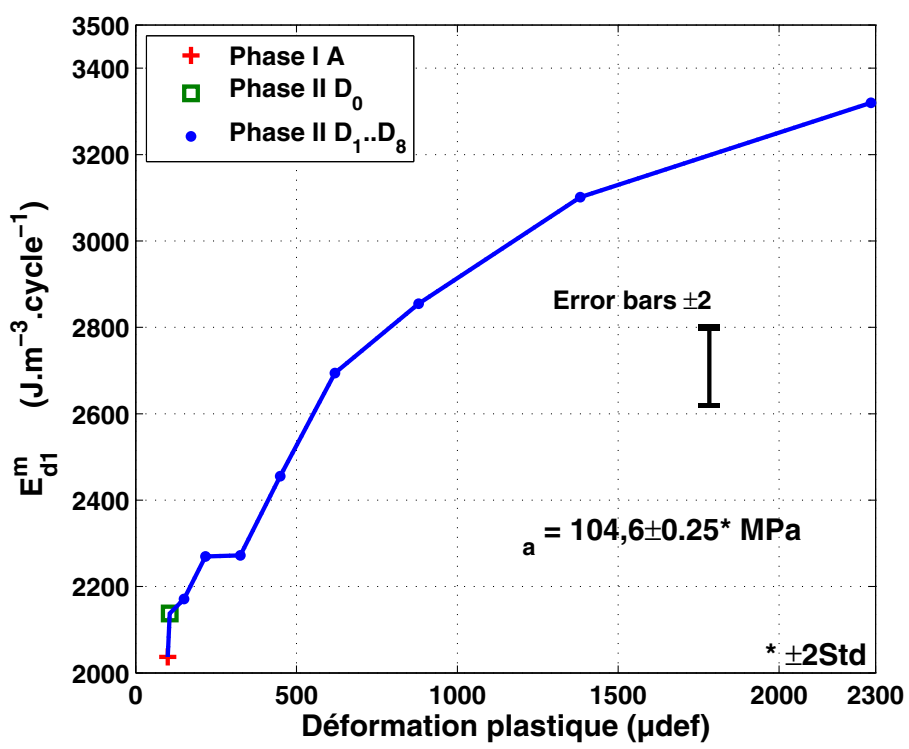

Fig. 4. Dissipative energy measurement $E_{d_{1}}^{m}$ at $\sigma_{\max }=104,6 \pm 0.25 \mathrm{MPa}$ versus plastic strain $\epsilon^{p}$.

\section{Conclusion}

The dissipative energy due to internal friction of a dual phase steel evolution with the plastic strain has been measured. First, it clearly appeared that the dissipative energy due to internal friction increases with the plastic strain. When no plastic strain occurs, the dissipative energy measurement is well reproducible on the same specimen. Thus, the measurement of the dissipative energy variation versus the alternate stress in the stress domain where no plastic strain occurs is a "signature" of the material.

Correlation of the DP600 dissipative energy signature during fatigue tests will be studied in future works.

\section{References}

1. H.F. Moore, J.B. Kommers, Chemical and Metallurgical Engineering 25, (1921) 1141-1144

2. G. Fargione, A. Geraci, G. La Rosa, A. Risitano, International Journal of Fatigue 24, (2002) 11-19

\footnotetext{
${ }^{1} \pm 2$ Standard deviation of the alternate stress $\sigma_{a}$.
} 
3. A. Chrysochoos, H. Louche, International Journal of Engineering Science 16, (2000) 17591788

4. F. Maquin, F. Pierron, Mechanics of Materials 41, (2009) 928-942

5. D. Caillard, J.L. Martin, Thermally activated mechanisms in crystal plasticity (Pergamon, Amsterdam London 2003) 85-123

6. A. Granato, K. Lücke, Journal of Applied Physics, 27:6, (1956) 583-593

7. T. Tanaka, S. Hattori, Bulletin of the JSME, (21:161) (1978) 1557-1564

8. C. Déprés, M. Fivel, L. Tabourot, Scripta Materialia, 58:12 (2008) 1086-1089

9. T. Fujii, C. Watanabe, Y. Nomura, N. Tanaka, M. Kato, Materials Science and Engineering A, 319-321 (2001) 592-596

10. P. Lukás̆, L. Kunz, Materials Science and Engineering A, 322:1-2 (2002) 217-227

11. H. Mughrabi, F. Ackermann, K. Herz, ASTM Special Technical Publication, 675, (1979) 69105

12. C. Doudard, PHD Thesis, ENS Cachan, (2004), in French

13. C. Mareau, PHD Thesis, ENSAM, (2007), in French 